\title{
Article
}

\section{Overseas internships as a vehicle for developing a meta-level awareness regarding science communication}

\author{
Kayoko Nohara, Michael Norton, Miki Saijo, Osamu Kusakabe \\ The overseas internship programme offered at Tokyo Institute of Technology as part of the science \\ communication curriculum is highly significant, as it prompts graduate students to acquire new skills \\ and awareness levels, including an enhanced meta-level understanding of the importance and complexity \\ of human communications. The capacity to correlate and respond on-site in human interaction can be \\ gradually cultivated during the internship as students experience diverse communication environments. \\ Moreover, the exposure to different organisational, cultural and social environments helps develop a \\ more international outlook. As a result of the initial experience described in this paper, TiTech has \\ adopted internships as an important part of the educational tool-kit to produce scientists and engineers \\ who can play an active role at the global level using their acquired technical knowledge and broad \\ practical capabilities.
}

\section{Objective and approach}

In 2005, Tokyo Institute of Technology (TiTech) ${ }^{1}$ launched a new graduate course entitled 'Science and Engineering Communication: Theory and Practice.' ${ }^{2}$ An internship option was included with this course, and has already produced meaningful results ${ }^{3}$ : participants acquired new skills and awareness levels, including an enhanced understanding of the importance and complexity of communications, a broadening of each participant's personal perspective, and a marked improvement in each person's ability to interact with others. In the light of these outcomes, the internship was extended in 2006 from a purely national one to include an international option.

The objective of this paper is 1) to report on the implementation of this programme as part of the science communication curriculum; and 2) to discuss the significance of the overseas internship programme from both theoretical and practical perspectives. Data collected through the evaluations and interviews on the internships will be analysed utilising relevant theories of linguistics and communication studies.

\section{Context}

\subsection{The contemporary significance of science communication in Japan}

More than 20 years have passed since the importance of science communication was first recognised in the West and its methodologies espoused (e.g. Royal Society, $1985^{4}$ ). In Japan, recognition in the public domain has been more recent, with 2005 being declared the 'Inaugural Year of Science Communication'5. It was from that point that nation-wide efforts at promoting science communication began in various regions throughout Japan. Science communication is an activity in which people of varied backgrounds interact through a variety of media, such as through dialogue. Unidirectional outreach efforts or educational attempts by specialists to 'enlighten' the general public and fill their perceived knowledge 'deficit' is no longer regarded as an effective way of promoting the public understanding of science, and Japan is no exception in this regard. ${ }^{6}$ The more recent emphasis is to 
engage in a collective effort with citizens, both directly and indirectly, to explore the interaction between society's needs and aspirations, and the possibilities and limitations of science. ${ }^{7}$

These activities come at a time when the impacts of science on society are becoming not just more substantial but more difficult to address in terms of public debate. For instance, science has identified the limits of the global environment and there is now broad recognition of the importance of 'sustainable development, ${ }^{8}$ and on specific aspects such as the debates on the Kyoto Protocol. Sustainable Development can be seen as addressing the conflicts between environmental damage and the activities of countries throughout the world in pursuit of efficiency and convenience. In order to achieve a more sustainable state, we must first ask what needs to be done both collectively and individually, and the answer to that question is something that we ourselves must craft. Essential to this process is to establish a method of satisfying the fundamental needs of humanity while maintaining fairness between generations in the long term and staying within environmental limits, as an alternative to acting primarily out of concern for immediate gain. This objective needs to be addressed socially, economically, ecologically, spatially, and culturally (1991 World Conservation Union), and is a good example of where the interaction between science and society is a critical component.

Another aspect of science communication is to allow citizens to communicate their needs and values from their own points of view. There is also merit in the science and technology community working to clarify the long-term future development of its various research agendas. It is particularly important for young scientists to be able to perceive the role of their research in the complicated global context of social, ethical and environmental aspects. In order to create a society in which 'the public adjustment of science and society ${ }^{9}$ occurs, a dialogue between scientists and the average citizen will be indispensable. In the present age when people tend to be isolated in their respective domains, living in an environment we call an 'information society', it is perhaps most desirable that this dialogue takes the form of an exchange between people that is characterised by its humanness.

\subsection{The dual layers of the science communication design}

The interactions described above sometimes take the form of a dialogue and, at other times, the form of joint participation in the same activity or event organised for the purpose of public understanding or engagement. Here we are not restricting ourselves to the stance that all conversations related to science should be included under the rubric of science communication. Instead, we characterise science communication as follows: 1) Topics are restricted to those related to science and technology; 2) Interactions occur between participants who have little knowledge or experience of science and those rich in knowledge; 3) A distribution of roles exists among the participants. ${ }^{10}$ Science communication is commonly characterised by the fact that it occurs in a context separated from ordinary life. Consequently, a certain intentionally constructed situational framework and a communication design are necessary. Moreover, it is here that we find the reason for more actively discussing and analysing the very methodology that should underlie science communication.

Science communication can be seen as shaped by a dual-layered communication design. One layer is comprised of the formatting done prior to the event and initiated from the position of the organisers of the interaction (this includes the preparatory role of the facilitator). The second layer is comprised of the diverse strategies and skills possessed by the individual participants ${ }^{11}$. In this paper, we will primarily focus our discussion on the second layer, namely the individual communication skills and the training needed to acquire such. The key question is what can be done at university level to assist in developing such abilities. Among the various educational programmes that have been put in place, the course in science communication implemented at TiTech is unusual in including internships and (to our knowledge) unique in Japan in offering overseas internships, which allow participants to learn through on-site experiences. 


\section{Course activities}

\subsection{The TiTech educational programme in science communication}

The curriculum of "Science and Engineering Communication: Theory and Practice" is divided into two phases (both are 14-15 weeks): 'Science communication 1' includes theoretical units, while 'Science Communication 2' offers classroom exercises, as well as practical training and skill development conducted outside the university. ${ }^{12}$ These activities include an internship, the planning and management of a café scientifique, or the creation of image contents or manuscripts that introduce science in a general way. The entire programme is comprised of three structural segments: 1) Assisting students in acquiring knowledge about science communication and its related theories; 2) Dispatching students on internships both inside and outside; and 3) Offering practical training based on the café scientifique.

\subsection{Dispatching students on internships}

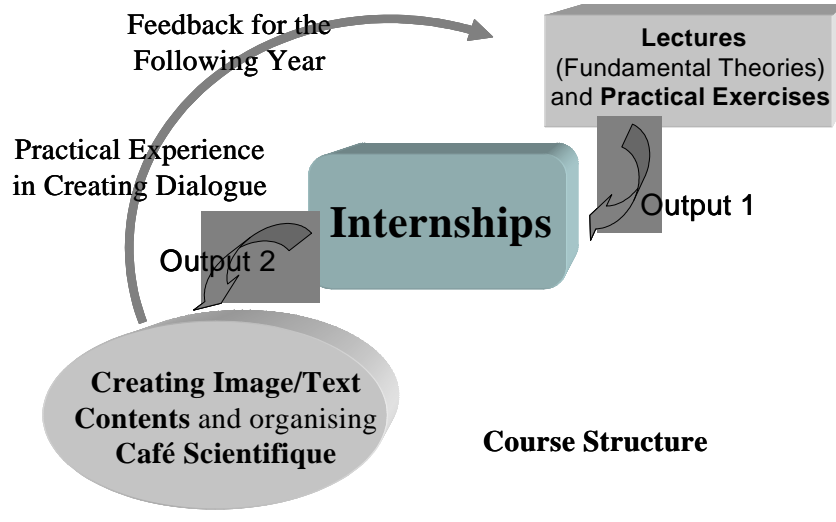

Under the internships, master/doctoral students ${ }^{13}$ are sent as interns for approximately 3 weeks (short term) or 3 months (long term) to various institutions, including media organisations (Yomiuri Newspaper Tokyo Headquarters, Nikkan Kogyo Shimbun Ltd., Nikkei Business Publications, Inc.), public policy institutes (Japan Science and Technology Agency [JST], National Institute of Science and Technology Policy [NISTEP]), and a science museum (National Museum of Emerging Science and Innovation [MIRAIKAN]), that serve as points of contact for science and society (23 students have been dispatched in the scheme so far [2005-2007]). The primary purpose of this programme is the cultivation of young scientists or engineers who are able to communicate at the various points where science interacts with society. It is also important, however, to include within our perspective the need to educate outstanding people who can join the workforce to engage in science communication activities in some form in the future --since science communication will most certainly grow in importance. For instance, in the Ubuntu Declaration adopted in 2002 at the 'World Summit on Sustainable Development', it was emphasised that 'it will be essential that we pay greater attention to the role of education and the scientific community'. Graduates of the course with experience and communication skills may see employment opportunities in such areas as general science writing, facilitating such events as a café scientifique, or in activities related to sustainable development such as those just mentioned. Effective programmes can be used, not only to educate students, but also to provide recurrent education for people who already work in science and technology fields. Our objective is that the programme designed by TiTech and experience gained therein will be of value in developing such human resources.

\subsection{The first trial of an Overseas Internship Programme 2006/7}

Overseas internships were established in 2006, initially in the UK where science communication has been established considerably longer than in Japan. In seeking the hosting organisations' agreement, we ensured they appreciated the main thrust of our programme: namely to assist students in science and engineering to acquire communication abilities and a more complete perspective regarding science and 
society. Organisations agreeing to act as hosts by allowing students to experience the actual work of science communication while abroad for approximately 2-3 weeks were the following in 2006:

1. The Dana Centre (Institution Associated with the Science Museum) ${ }^{14}$

Intern Profile (1 student): first-year masters student, male, majoring in Environmental Politics. Work:

- participated in various events including the Dana Centre café scientifique, Dinner@Dana, etc.;

- research and planning for future events.

2. Science in Society Team at the British Association for the Advancement of Science (BA) ${ }^{15}$ Intern Profile (1 student): second-year masters student, female, majoring in Management of Technology.

Work:

- assisted with the preparation and implementation of the Festival of Science in Norwich.

3. Parliamentary Office of Science and Technology (POST) ${ }^{16}$

Intern Profiles (2 students): second-year masters student, female, majoring in International Development; third-year doctoral student, male, majoring in Environmental Engineering.

Work:

- participated in various events, seminars and meetings the POST organised to inform public policy regarding science and technology and to offer this information for parliamentary debate.

4. Royal Society ${ }^{17}$

Intern Profile (1 student): first-year doctoral student, male, international student from Cambodia, majoring in Civil Engineering.

Work:

- helped the planning for summer science exhibition 2007;

- involved in searching for fund support for the scientific book prizes project;

- helped with media training course for scientists.

In order to observe events occurring during the internships and following the flow of the resulting ideas and experiences, we utilised "XOOPS," a software system for mailing lists and content management. This not only allows student monitoring by the instructors, but also facilitates an information exchange within the group of students participating and between students and staff. In "Science Communication: Theory and Practice," we set up a website with a groupware function, allowing users to share various data, such as the class syllabus and schedule, and data from the class. The website includes a bulletin board to allow the internship students, other students, instructors, and others connected to the project (teaching assistants, office support staff etc.) to freely contribute diary entries and personal impressions, and file questions and answers. While the students are not tied to a fixed and arbitrary reporting frequency, they are instructed to report periodically "what they discovered during their activities on a particular day," "the issues they need to convey to the office," etc.

After introduction, use is not immediate but students gradually use it more frequently. With engineering students, there seems to be a great deal of significance associated with setting up a system based on the use of IT due to their routine use of IT support systems, even when they are outside of class or away from their work at the internship. While the specific content of the work at the internship cannot be shared since we adopted a confidentiality agreement with the hosting site regarding the individual work content (and as a result this limits students' ability to get concrete advice and express detailed opinions), the staff are able to know in real time about the new situations in which the students find themselves, as well as their thoughts, worries and how they have managed their difficulties (TiTech Science Communication Office 2006). This bulletin board thus seems to have functioned as an effective means of understanding the growth process of the students, through which they discovered strategies to overcome the obstacles which they encountered.

\section{Expected benefits of the Internships}

Let us first discuss what positive effects common to both domestic and international internships can be expected in theory. 


\subsection{Encountering new communication patterns}

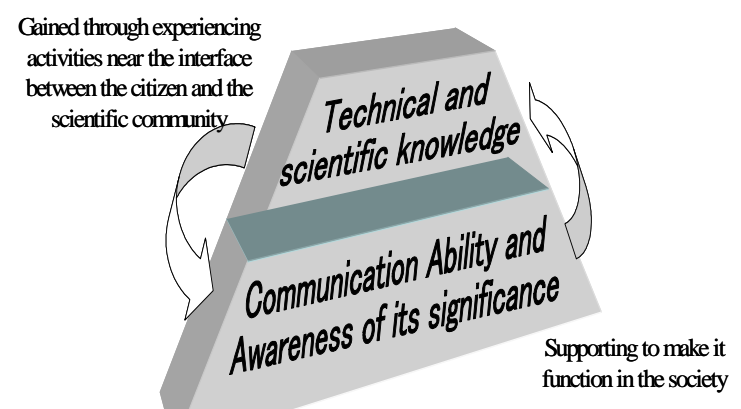

Communication Ability as a Supplementary Skill

The internship model we have introduced differs from both internships based on employment experience (which tend to be part of job-hunting activities), and internships designed to acquire specific technical knowledge or skills (which are often used in Japanese engineering organisations). In contrast, our internship aims specifically to assist students to learn the importance of communication, and to develop the necessary skills and outlook, through practical experience of activities near the interface between the average citizen and the scientific/technological community. In short, this internship can be characterised as acquiring 'backup skills' - by cultivating communication ability as a supplementary skill which can assist in further activating the technical knowledge one already has as a scientist. The relationship between the activities and the skills acquired in internships of this type can be illustrated as the diagram:

Communication occurs continuously during the routine of science and technology research - for example, discussion at a conference, getting instructions from a teacher, debates related to joint research, and everyday conversation between students in a laboratory. We label this 'intra-science-community communication'. Research students' everyday experience is primarily within their intra-science-community communication. In this environment, there exists a common language usage, norms or social dialect with which participants are familiar, and these conventions serve as the context in which judgments are made. In contrast, during internships, students may well have to interact with participants whose discourse is quite unfamiliar to them. This more diverse mode of communication can be called inter-community communication'. In the latter, a number of problems can result from differences in communication norms, and there are no (or few) standards for interpretation that can provide a mutual foundation for understanding. Through the internships, students are expected to encounter a variety of unexpected situations with potential for miscommunication, and to increase their capacity to cope with inter-community communication only gradually. In fact, after the dispatch, we found evidence that the students do not simply experience inter-community communication but also show some signs of acquiring meta-level awareness of communication through such interactions. This point will be touched upon in discussing the result in 6.1.

\subsection{Broadening knowledge and outlook}

Another major benefit expected from dispatch is that students may acquire broader perspectives through encountering systems which are substantially different from those with which they are already familiar. Overseas there are differences in the political framework within which organisations function, and they may well play very different roles compared with Japan. In addition, workplace practice, roles and underlying assumptions about behaviour can be quite different. Thus, while language may appear to be the greatest single difference between national and overseas internships, there are many other opportunities to learn (or be distracted by) differences in the organisational and social environment encountered.

For instance, the UK organisations receiving interns have similarities to but are not analogous to Japanese organisations. Thus, the Royal Society shares some functions with the Science Council of Japan, but has a much broader responsibility in terms of policy advice and in funding research. POST is a Parliamentary body which has no equivalent in Japan - it serves the whole Parliament (both upper and lower houses), focuses entirely on political and policy issues related to science and technology developments, and serves equally governing and opposition interests. The BA has some common functions with that of JST, but is independent of government and essentially a private organisation. Finally the Dana Centre has no analogue in Japan but has close links to the Science Museum which has similar functions to the Miraikan. In general all these organisations have a much longer history than in those in Japan - the Royal Society was formed in 1660, the BA in 1831, Science Museum in 1857 and only POST (1989) and Dana Centre (2003) are recent creations. 
Through the internships, the interns thus have an opportunity to learn not only about another country's response to the same subject of science communication, but the political and policy framework within which that policy is pursued. At the same time, the intern is forced to evaluate the equivalent Japanese structures to create a basic frame of reference into which they can place their new experiences. Information exchange with the domestic interns through Xoops encourages such considerations.

\section{Method of evaluating the internship results}

In order to assess the results of the overseas internships and discuss their significance, we collected data through the following:

a) Written questionnaires;

b) Interns' comments from individual and group interviews;

c) Intern supervisors' comments by e-mail.

We attempt to draw some tentative conclusions regarding dispatch outcomes and the significance of the internship from a qualitative analysis of the data. The results we have achieved thus far are based simply on the four receiving institutions above and on the experiences of the five students sent on overseas internships during 2005. Some data from each category will be demonstrated later in Section 6.

a) The questionnaires included a self-evaluation by each intern before and after the internship, and one made of the intern's performance by the intern supervisor at the receiving institution; again both before and after the internship. The "before internship" evaluation was made at the end of the first day of the internship, and the "after one" was at the end of the last day. The evaluation sheets contained 25 criteria which could be categorised according to the 5 primary considerations shown in the diagram below. ${ }^{18}$ Performance in each of the criteria was graded according to ABCDE scale (Excellent to Poor), which count 5-1 points.

\section{Criteria for Evaluations}

\begin{tabular}{|c|c|}
\hline 1 & $\begin{array}{c}\text { Familiarity with the types of work at the internship site } \\
\text { (media organisation, public policy institution, museum) }\end{array}$ \\
\hline 2 & Improvement in the skills necessary for the work \\
\hline 3 & Aware of the social impact on science and technology research \\
\hline 4 & Clear about own career goals \\
\hline 5 & Grasps the job flow at the internship site \\
\hline
\end{tabular}

(b) Interns' comments from individual and focus group interviews We held individual and group interviews with the interns within 1-2 weeks of their return, using a set of questions prepared by the project staff. Interviews followed the format: 1) the same subject items were used for each interview; 2) all members were asked to respond; 3) it was acceptable for other members to comment freely during the session; 4) all could take notes. The following seven points were established as the items to be covered in the interview.

Items for the Focus Group Interview

\begin{tabular}{|rl|}
\hline A & Internship Experience \\
\hline 1 & The internship experience and the overall impression \\
2 & Significance of what you experienced at this point in time \\
3 & Did your way of looking at media/public policy institution/museum change? If it did change, \\
& how did it change? \\
4 & How do you think this experience will influence you personally hereafter? \\
\hline B & Science Communication in General \\
\hline 1 & What do you think is science communication? \\
2 & Significance of the internship in terms of science communication \\
3 & How would you think of the internship in terms of science communication theory?
\end{tabular}


(c) Intern supervisors' comments

We also received comments from the interns' supervisor from the perspective of the host institution - either together with the questionnaire (a) or in separate e-mails after the internship.

\section{Data obtained and analysis}

In this section, we consider some of the data obtained from the above evaluations and analyse the positive results gained by the dispatch.

First, let us present the results of evaluation (a) (we intend to accumulate questionnaire data over the coming years before submitting the data to quantitative statistical analysis).

\begin{tabular}{|c|c|c|c|c|}
\hline \multirow{2}{*}{$\begin{array}{l}\text { Evaluation } \\
\text { Main Items }\end{array}$} & \multicolumn{2}{|c|}{ Students } & \multicolumn{2}{|c|}{ Receiving Institutions } \\
\hline & Start & Completion & Start & Completion \\
\hline $\begin{array}{l}1 \text { Familiarity with media, } \\
\text { public policy institution, museum }\end{array}$ & 3.27 & 4.70 & 3.53 & 4.73 \\
\hline $\begin{array}{l}2 \text { Improvement in the necessary skills } \\
\text { for the job }\end{array}$ & 3.56 & 3.83 & 4.09 & 4.23 \\
\hline $\begin{array}{l}3 \text { Recognition of the social impact } \\
\text { of the research }\end{array}$ & 3.62 & 3.53 & 3.78 & 4.07 \\
\hline 4 Clarity of the student's own career path & 3.33 & 3.88 & 3.73 & 3.70 \\
\hline $\begin{array}{l}5 \text { Understanding of the work flow } \\
\text { at the institution }\end{array}$ & 3.33 & 4.26 & 3.29 & 4.17 \\
\hline
\end{tabular}

Chart 1. Questionnaire (a).

Combining the results of evaluation (a) above with the results of (b) Interns' comments and (c) Intern supervisors' comments, we analyse the overall results under the three primary categories of: A. linguistic and cultural challenges; B. acquiring knowledge of institutions; and C. ability to assess science communication policies and discuss them.

\subsection{Meta-level thinking about communication and the capacity to respond on-site}

As expected, the students experienced a variety of inter-community communications, which were new to them, during the internship. The psychological pressures associated with human interactions in a new context with a new character are immense. Chart 1 shows that with regard to the statement "Improvement in the necessary skills for the job," the students responded with a uniformly low evaluation compared to the receiving institutions at both the start and end of the internship. The students appeared to be aware of their lack of skill in having a smooth conversation, nor did they have the skills to speak and write in an easy-to-understand manner. They were also very much aware of the frustrations associated with not being able to give answers to questions easily, even though they had the required knowledge (The BA intern, individual interview, 2006). This indicates that they were beginning to "notice" the complexity and depth involved in communication with unfamiliar people.

The fact that the content of the internship was not directly related to their technical research field where intra-science-community communication functions smoothly, but was work connected to science communication prompted them to assume a passive role. Their lack of confidence is somewhat similar to what we see when a person begins speaking to a specialist with the expression 'I'm just a lay person anyway' (The DANA centre intern, students group interview, 2006). Three of the overseas interns spoke not just about the difficulties of English as a language, but the difficulties of the communication process itself; they spoke at the meta-level about significance and the sense of achievement associated with moments when they communicated well. In short, they returned with a full awareness that they had 'thought and learned' about the communication process. 
The fact that the internship experience is all in English makes inter-community communication even more challenging for them. "The lack of confidence I suffered from came largely from the linguistic and cultural gap" (The Royal Society intern, individual interviews, 2006). Thus the lack of full command of English and of a full understanding of British culture added a great deal of insecurity to the intern's psychology. According to Chart 1, two students evaluated the degree of their development at the close of the internship as poor, and compared to their evaluation of the first day, there was only modest change (the evaluation went from ' $C$ ' to ' $B$ ' or ' $D$ ' to ' $B$ ') ( $v d$. 'ABCDE scale' described in 5). The students seemed to be especially aware of their inability to use English as a medium of communication. They could not easily find the clues that would allow them to communicate more smoothly and this caused frustration. Though prior to being dispatched, the DANA Centre intern had received high marks on the TOEIC (890 points), he became fully aware that this did not translate into listening ability when he was in the position of having to communicate in a fresh context (individual interview, 2006).

Such weaknesses are related to correlating and responding abilities, which are the general skills in handling the question of how to interpret a piece of utterance and how to respond in each situation. Such ability is one that can be 'noticed' and 'cultivated' gradually as the student experiences different communication challenges. The DANA Centre intern "started to become increasingly aware that the possibility of relevant information being included in the communication somewhere is high", and started to intentionally look for it (individual interview). This realisation is highly significant. Overseas internships provide students with the possibility of recognising the importance of correlation and the capacity to respond on-site. In addition, it is essential for young scientists to acquire an ability to use English as a lingua franca in scientific information exchange and this can be strengthened through the internship experience.

\subsection{Broadening knowledge of institutions and the social context}

Chart 1 shows that both the students and the receiving institutions gave very high marks to "Familiarity with media and public policy institutions" at the completion of the internship, a marked difference from the start. It became clear that the students had raised their awareness of various types of work and institutions which they had found difficult to conceptualise before embarking on the internship. This all occurred in a short period of time. When the dispatch started, the interns had (or at least THEY SAY they had) a low self-evaluation. It was especially interesting to learn about their insecurities regarding problems surrounding the interface between their own research field and society in general, and their knowledge about the internship locations, which were outside of their intra-science community. In addition workplace practice, roles, and underlying assumptions about behaviour can be quite different. Thus, although at first sight language may appear to be the greatest single difference between national and overseas internships, there are many other differences in the organisational and social environment which provide opportunities to learn (as well as more potential barriers to be overcome). The social context of the internships may thus also have educational value. The balance between individual and group working; the way in which work is apportioned and distributed through the management structures differ between countries. The UK workplace lacks the hierarchical and respect structures of many Japanese organisations. Participants are expected to contribute their ideas spontaneously regardless of gender, age or seniority; "I was also surprised that a staff that was about the same age as myself had full responsibility for an event. I too wanted to try and have that same kind of mature sense of responsibility'. (The Dana Centre intern, individual interview, 2006). The intern thus observes a more open and less structured frame for communication and interaction, and this poses challenges to him/her to become more proactive than might be the case in a Japanese organisation. Outside working hours opportunities for travel and socialising also contribute to a broadening of outlook and ability to deal flexibly with different social scenarios and situations. The internship provides a broadening experience contributing to internationalisation of outlook, as well as the ability to recognise and adapt to alternative models of behaviour. Since many of TiTech graduates join international companies this may be an additional useful asset. 


\subsection{Acquiring an ability to assess science communication policies and associated attitudes}

The opportunity to learn the political and policy framework within which science communication policy is pursued, forces the intern to evaluate the equivalent Japanese structures. Overseas organisations are likely to be established on different principles to their Japanese equivalent, so this leads the intern to consider broader issues such as policy goals, resources and implementation strategies, creating a base from which the underlying assumptions underpinning Japanese practice can be considered.

For instance, whether a science advisory service could operate within the Japanese Parliament; whether interactive models of engagement are suited to Japanese culture, and so on. With such diversity, interns thus come back with a more comprehensive 'toolbox' than they would obtain from an internship within Japan, which can facilitate in transferring innovative approaches and new ways of thinking to science communication within the Japanese context. "I came in contact with the actual state of science communication in the UK and encountered a high level of interest among people. I learned about having different views, having the right to know, and asserting ones opinion. [...] I encountered things I couldn't learn about when talking with Japanese, and came in contact with a fascinating culture, value system, and world of ideas. I wished that I could understand those in the UK better and convey to them my thoughts more accurately. These frustrating feelings will serve as a strong incentive to study English. I began to see that a great many future jobs will be available for scientists who can communicate and science communicators and I realised that people who can serve as role models for scientists will be much needed"(The BA intern, individual interview, 2006).

Additionally the students encountered the frustration of not being able to solve the problems of science communication based solely on their technical knowledge. What is particularly fascinating is that many students after the internship said, "I realised that there is no manual or set answer to the question of how a skilful compromise can be crafted between society and technology" (BA intern 2005, individual interview). When an overview is done of the results, and the self-evaluations and the external evaluations are compared, we discovered that the students were prompted to adopt a perspective that relativises their own research within the framework of society and its policies, and they were also prompted to notice and think about the importance of that relativisation.

\section{Conclusion}

\subsection{Programme assessment}

From the results above, we conclude that the overseas internships are fulfilling their intended primary function of nurturing the communication ability of young researchers. However there are more detailed conclusions which can be drawn as well as points for improvement in future implementation.

First, it is clear that one needs knowledge about linguistic, social and behavioural norms in order to utilise linguistic systems fully in real life ${ }^{19}$. Thus if someone wishes to communicate effectively in an English-speaking environment, they need to know how people behave there. This will help provide the capacity to correlate and respond on-site in the appropriate context. Such abilities can be obtained by exposure to diverse situations, which may lead to a heightened awareness about communication.

Secondly, exposure to different organisational, cultural and social approaches helps develop a more international outlook, which can also act as a trigger for innovative thinking in Japan. The students who experienced science communication in a different context abroad, introduced the idea of the "necessity of relativising technology". In the modern era in which we are seeking the elusive goal of sustainable social development for our world, the question of crafting a compromise between technology and society is an issue that is being debated and grappled with globally. It goes without saying that the students who have thought about and experienced science communication from this broader perspective will have the potential to drive forward the debate and make an effective contribution to related activities that have just begun to take place in Japan. 


\title{
7.2 Implications for future internships
}

Experience with the first year's programme also causes us to review aspects of the internship management. First of all, it is difficult to hold external internship programmes at a fixed period of time during the academic term because of laboratory and job-hunting activities. Therefore it will be necessary to think about shifting the winter internship to spring/summer vacation.

Another issue relates to the IT-based monitoring system. At present there is some conflict between the wish for project staff and teams of students to efficiently share the various student's experiences and learn together as a group, and the need to respect the confidentially commitments given to the receiving institutions. Using the networked IT system also places a burden on interns who may simply be too busy to report frequently what they did on each day. A less stressful method for the interns needs to be considered.

Experience from the first series of internships suggests that the higher-level learning opportunities can only be grasped when the intern has not only adequate English knowledge but also sufficient selfconfidence to use it. In the relatively short period of the secondments, it is unlikely that the intern's basic ability in English will substantially improve, thus the key selection criteria remains ability before secondment. Initial experience also suggested that better preparation on the background and functioning of the hosting organisation will speed up the intern's learning processes during the secondment. Future internships will thus be screened according to the following:

1. Stricter screening of actual ability to understand and engage in English conversations. This is a request emphasised by many host institutions. ${ }^{20}$

2. A requirement to research the rationale and working methods of the hosting organisation before secondment and to produce an initial assessment comparing it with Japanese equivalent organisations. This provides a frame of reference which should be useful during the internship.

3. A requirement after the internship to critically analyse the system of science and society in the foreign country and compare it with Japanese practice. ${ }^{21}$

Finally let us present an evaluation from David Cope, the Director of POST. This evaluation reviewed our whole programme including the overseas internship as an educational programme focusing on science communication and conducted by an institution of higher learning:

\begin{abstract}
From my experience with the UK (science communication) schemes, and based on the description provided by TiTech, it would appear that their(TiTech) science communication course has similar objectives and that the structure appears wholly appropriate to those objectives. The course is however unusual in attaching the communication module to an existing engineering master's course. In the UK, such courses are often presented as simple media training over one to three days. I believe the TiTech approach of a more thorough grounding in the theory and objectives of science communication, combined with real practical experience is unique and undoubtedly valuable. The fact that the course has also integrated a science cafe approach into the same module is also, as far as I am aware, unique.
\end{abstract}

As this encouraging comment suggests, the Titech internship programme is helping to produce scientists with the technical knowledge, broad outlook and practical capabilities needed to play an active role at the global level at the interface between science and technology and Society. The need for a new way of working based on a dialogue between the technical science community and our general society is becoming increasingly recognised within the international community, as we recognise the importance of developing sustainable technology, economies and societies. We intend to continue to improve the Titech programme further in the future towards fulfilling this need.

\section{Notes and references}

\footnotetext{
${ }^{1}$ Tokyo Institute of Technology is the leading higher educational and research institution in Japan specialising on the training of science and engineering students.

${ }^{2}$ With funding from the JST programme.
} 
${ }^{3}$ Osamu Kusakabe, Kayoko Nohara, Miki Saijo, Aki Higashii (2006) 'The Role of internships based on industry-public sectoruniversity collaboration', Kogaku kogyo kyoikukenkyu koenronbunshu, Japanese Society for Engineering Education, pp. 258-259.

${ }^{4}$ Royal Society (1985) The Public Understanding of Science, Royal Society, London.

${ }_{6}^{5}$ Mamoru Hayashi (2003) NPO Science communication news, http://scicom.jp/.

${ }^{6}$ Esuke Enoki (2006) NPO Science communication news, http://scicom.jp/blog/2006/.

${ }^{7}$ As part of the efforts to strengthen science communication practice in Japan, the Ministry of Education, Culture, Sports, Science and Technology (MEXT) has allocated funds to establish courses in universities, and research grants to develop models for promoting the activities of researchers and communicating related information; these funds are allocated by the Japan Science and Technology Agency (JST). See their activities in promoting public understanding of science and technology, http://rika.jst.go.jp/outreach/top.htm.

${ }^{8}$ Development that 'fulfils the needs of today's generation, without impeding the ability of future generations to satisfy their needs' (1987 United Nations 'World commission on environment and development').

${ }^{9}$ Hideyuki Hirakawa (2006) 'Biotechnology no kokumin rikai', Kaisei Report, http://www.life-bio.or.jp/topics/.

${ }^{10}$ Miki Saijo, Kayoko Nohara, Osamu Kusakabe (2007) 'Danwakenkyu kara mita kagakugijutsu communication no igi to jissen', Kogakukyoiku 55-1, pp.59-65.

11 vd. 'techniques in negotiation' in Hiroshi Kimura (1998) Kokusaikoshogaku, Keisoshobo: Tokyo, pp. 23-40.

12 The course participants' number varies between 20-50 in lectures and 10-15 in practical exercises by semester. We suggest up to 30 as the maximum capacity in both. It needs to be noted that two professors and one full-time researcher manage the course throughout the year with assistance of a number of guest lecturers and speakers.

${ }^{13}$ It is desirable that the students already have steady knowledge and technique in their specialised area with which they can start practicing science communication activities. Thus we suggest the programme is of much use for master students or above.

${ }_{14}$ The Dana Centre promotes public dialogue regarding science and technology. During the day, it offers a coffee bar and Internet café so that visitors to the museum can easily drop by; in the evening, the Centre turns into an event center with regularly scheduled events, such as café scientifique and other events to stimulate dialogue with the public on science related issues, http://www.danacentre.org.uk/.

15 The BA is a government-supported NPO promoting the public's understanding of science and engineering. For example, in September of each year, it sponsors The Festival of Science, which is the largest public technology event in Britain. Moreover, it organises the media fellowship scheme to help scientists, social scientists, and engineers understand how the media deals with science and technological issues. For a period of 3-8 weeks, the fellowship recipients work alongside newspaper, broadcast and internet journalists, learning how to convey the newest information in science and technology, and experiencing this kind of work first-hand (British students are the only recipients of this fellowship), http://www.the-ba.net/the-ba/index.html.

${ }^{16}$ The POST conducts the investigations necessary to inform public policy regarding science and technology and provides this information to parliament. It publishes various "POST NOTES" (short briefing notes) and other investigative reports, in addition to offering briefings and related materials to various Parliamentary Committees,

http://www.parliament.uk/parliamentary_offices/post.cfm.

${ }^{17}$ The Royal Society is the UK's national academy of science. Along with its grant funding and professional activities, it has sections devoted to the interaction of science and society which participate in the shaping of public policy related to science and technology. Since 1985, it has supported a range of programmes focused on public understanding and engagement with science and technology, http://www.royalsoc.ac.uk/.

${ }^{18}$ These are based on the standards the BA is using for its own evaluations within the various schemes for media internships for scientists, etc. vd. TiTech Science Communication Project (2006) A New Attempt by Tokyo Institute of Technology - Science and Engineering Communication: Theory and Practice. Tokyo Institute of Technology.

${ }^{19}$ Hayashi Susumu (1985) Communication-ron, Yuhikaku: Tokyo, pp. 265-66.

${ }^{20}$ A pre-dispatch intensive English course in talking about scienctific subjects might be helpful for the participants.

${ }^{21}$ Thus the interns for POST were able to take part in an official meeting between POST and the Research Office of MEXT associated with Japan's Upper House - to get a general view of the differences evident in the organization and activities of Japan's institutional counterpart.

\section{Authors}

Kayoko Nohara received her D. Phil in Translation Studies from University of Oxford. She worked in the Faculty of Oriental Studies at University of Oxford, Gakushuin Univeristy, Katholieke Universiteit Leuven, Belgium, and is currently an Associate Professor at Tokyo Institute of Technology, teaching Linguistics, Japanese and Science Communication. Recent publications include: On the Strategic Use of Proper Names in Popular Japanese Literature", in M. Garant ed. Current Trends in Translation Teaching and Learning, Helsinki, 2006. E-mail: knohara@ ryu.titech.ac.jp.

Mike Norton is Professor at the Innovation Management Institute, Shinshu University, with a PhD in Chemistry from Bristol University. Mike worked for the UK Government on environmental protection and biotechnology research and policy. He was director of the Parliamentary Office of Science and 
Technology in London and Counsellor in Science and Technology for the British Embassy in Tokyo.

E-mail: norton@im.shinshu-u.ac.jp.

Miki Saijo is professor at the International Student Center at Tokyo Institute of Technology, with a $\mathrm{PhD}$ in Applied Linguistics from Ochanomizu University. Her research focuses on language asymmetries in dialogue between scientists and non scientists. E-mail: msaijo@ryu.titech.ac.jp.

Osamu Kusakabe is Professor at the Department of Civil and Environmental Engineering (School of Science and Technology) at the Tokyo Institute of Technology, with a PhD in Engineering from the University of Cambridge. His research focuses on geotechnical engineering and engineering education. E-mail: kusakabe@cv.titech.ac.jp. 